\title{
HEIDEGGER Y BULTMANN. EN EL PRINCIPIO ERA LA EXÉGESIS ${ }^{*}$
}

\author{
HEIDEGGER AND BULTMANN. IN THE BEGINNING \\ WAS THE EXEGESIS
}

\author{
Prof. Sylvain Camilleri² \\ Instituto Superior de Filosofía \\ Universidad Católica de Lovaina, Bélgica
}

\begin{abstract}
Resumen: El presente estudio es un intento de restaurar el significado y el valor de las "Escrituras" en el corazón de la relación dialéctica entre fenomenología y teología. Mientras que las Escrituras desempeñan un rol tremendo dentro de la vida religiosa como un catalizador, la filosofía de la religión tiende a evitar la exégesis por supuestamente ser una ciencia positivista alejada de la esencia de la religión. En este ensayo me gustaría contrarrestar tal punto de vista prestando especial atención a un evento histórico que se ha descuidado hasta ahora, es decir, el encuentro entre Heidegger y Bultmann en Marburgo, y en especial la primera contribución de Heidegger en el Seminario de Bultmann sobre el Nuevo Testamento. Esta contribución de Heidegger trata de las Cartas de San Pablo a los Romanos y ofrece un estimulante relato fenomenológico de la fe bíblica. Si bien este relato constituye un invaluable suplemento para los cursos magistrales de 1920/21 sobre el corpus paulinum en la Introducción a la Fenomenología de la religión, también da testimonio del hecho de que las Escrituras son esenciales para la comprensión de la dimensión performativa de la experiencia religiosa.
\end{abstract}

\section{Descriptores: Heidegger · Bultmann · Fenomenología $\cdot$ San Pablo $\cdot$ Exégesis}

\begin{abstract}
The present study is an attempt at restoring the significance and the value of "Scriptures" at the heart of the dialectic relationship between phenomenology and theology. Whereas Scriptures play a tremendous role within religious life as a catalyst, the philosophy of religion tends to avoid exegesis for purportedly being a positivist science far away from the essence of religion. In this essay I would like to counter such a view by paying close attention to an historical event which has been neglected so far, that is the encounter between Heidegger and Bultmann in Marburg, and more especially Heidegger's very first contribution to Bultmann New Testament Seminar. This contribution of Heidegger deals with Saint Paul's Letters to the Romans and offers a stimulating phenomenological account of Biblical faith. While this account forms an invaluable supplement to the 1920/21 lecture courses on the corpus paulinum in the Introduction to the Phenomenology of Religion, it also testifies to the fact that Scriptures are essential to the understanding of the performative dimension of religion experience.
\end{abstract}

Keywords: Heidegger $\cdot$ Bultmann $\cdot$ Phenomenology $\cdot$ Saint Paul $\cdot$ Exegesis

\footnotetext{
1* Traducción del francés de Jimmy Hernández Marcelo.

${ }^{2}$ E-mail: sylvain.camilleri@uclouvain.be
} 


\section{INTRODUCCIÓN}

Me gustaría defender aquí que las relaciones entre fenomenología y teología, ya centenarias, se han construido sobre un olvido. Desde la conferencia "Fenomenología y teología" (GA 9) 3 pronunciada por Heidegger en 1927 -en Tubinga y después en Marburgo- nos hemos acostumbrado a oponer fenomenología y teología, no como dos visiones del mundo, a la vez familiares y enemigas, sino como dos "ciencias" (en el sentido noble) que se caracterizarían por objetos distintos, esto es, el ser y -el ente- Dios, respectivamente. La teología es, entonces, reconducida por Heidegger hacia el positum de la fe -la fe cristiana engendrada por la revelación de Cristo, el Dios crucificado. El problema es que esta reconducción ignora por completo la mediación a través de la cual Cristo realmente se revela, es decir, la Escritura. Esto es aún más sorprendente ya que Heidegger, unos años antes, había dedicado extensos desarrollos a esta mediación bíblica, de la que hacía inicialmente el corazón mismo de la teología.

Este olvido de la exégesis -que podría considerarse como una "tercera ciencia", a medio camino entre la fenomenología y la teología, en la medida en que, por un lado, no presupone necesariamente la fe y que, por un lado, pregunta por la facticidad de una existencia (tocada por la gracia) y sus modos- ha condicionado todo el desarrollo de las relaciones entre la fenomenología y la teología, desde los préstamos de Bultmann a la analítica existencial del Dasein, que algunos de sus principios están integrados en una teología exegética que no cuestiona el medium que es la Escritura en tanto medium, hasta el "giro teológico" de la fenomenología francesa-denunciado por Dominique Janicaud- que se cuida de no definir el estatuto fenomenológico de las Escrituras y que incluso parece mantenerse deliberadamente apartado, al preferir estar cerca de las cumbres abstractas de la metafísica en lugar de sumergirse en los abismos concretos de la experiencia vivida a la que se refieren los textos bíblicos.

\section{HEIDEGGER y BULTMANN: PRIMER ENCUENTRO}

En este ensayo, en lugar de pensar en el olvido de la exégesis en sí mismo o por sí mismo, mi objetivo será desenterrar un momento concreto de las relaciones entre fenomenología y teología donde la exégesis es todavía el objeto del pensamiento y el objeto que da lugar al pensamiento, tanto para Heidegger como para Bultmann, quienes son los dos pilares fundadores de estas relaciones. En lugar

\footnotetext{
${ }^{3}$ Las referencias a las obras de Heidegger se harán directamente de la versión alemana Heidegger Gesamtausgabe (Frankfurt am Main: Klostermann, 1975-ss). Las citas se introducirán con las siglas GA -como abreviatura de Heidegger Gesamtausgabe- y el volumen del mismo; finalmente, añadimos el número de página, en los casos en los que la referencia lo exija.
} 
de partir del famoso curso dado por Heidegger en la Universidad de Friburgo en el semestre de invierno de 1920-1921, "Introducción a la Fenomenología de la Religión" (Heidegger, 1995b; GA 60), un curso del que he hablado extensamente en otro lugar (Camilleri, 2017), me apoyaré en un episodio "intermedio", a saber, la primera participación de Heidegger en el seminario sobre el Nuevo Testamento de Bultmann, pocos meses después de su llegada a Marburgo. Este episodio parece particularmente elocuente, ya que es equidistante, por un lado, del curso "Introducción a la fenomenología de la religión”, al que se acaba de hacer referencia y, por otro lado, de la conferencia "Fenomenología y Teología", pronunciada en 1927, año de la publicación de Ser y Tiempo (Heidegger, 1977; GA 2), la cual tendrá un profundo impacto en el pensamiento de Bultmann.

$\mathrm{Si}$ las circunstancias exactas del primer encuentro entre Heidegger y Bultmann no están documentadas, los testimonios directos e indirectos sobre los inicios de su colaboración son bastante numerosos. Sabemos que Heidegger participó regularmente en dos Graeca organizados por Bultmann, uno para los profesores y el otro para los estudiantes. ${ }^{4}$ También sabemos que por propio interés los dos hombres se reunían una tarde por semana para leer el Evangelio de Juan. ${ }^{5}$ Sin embargo, estas reuniones fueron precedidas por la participación de Heidegger en el seminario sobre el Nuevo Testamento de Bultmann del semestre de invierno de 1923-1924 dedicado al tema Die Ethik des Paulus. ${ }^{6}$ Puede incluso que los encuentros fueran el resultado de estos seminarios, ya que fue en este seminario cuando el teólogo escuchó por vez primera al filósofo y tuvo sus primeras impresiones sobre él. ¡Y qué impresiones! Bultmann se confiesa casi de inmediato a Hans von Soden, que estaba a punto de integrarse a Marburgo, así como a Friedrich Gogarten. Escribe al primero: "Esta vez, el seminario es particularmente instructivo porque participa nuestro nuevo filósofo Heidegger. Él viene del catolicismo, pero es enteramente protestante". ${ }^{7} \mathrm{Y}$ al segundo le dice: "Heidegger participa en el seminario y entre los estudiantes se encuentra una serie de personas de mucha valía, por lo que tengo la impresión de que

${ }^{4}$ Véase la correspondencia entre Martin Heidegger y Rudolf Bultmann (Heidegger \& Bultmann, 2009, pp. 24, 62, 69, 80, 84, 98, 111, 114, 122, 140, 141, 142, 143 y 204). Véase igualmente (Gadamer, 1977, p. 38).

${ }^{5}$ Véase la carta de Martin Heidegger a Karl Jaspers del 18 de junio de 1924: "La única personalidad $<$ de Marburgo >: el teólogo Bultmann, con quien me reúno cada semana" (Heidegger \& Jaspers, 1990, p. 23). Véase también la carta de Rudolf Bultmann a Friedrich Gogarten del 19 octubre de 1924: "Además, una tarde por semana leo con Heidegger el Evangelio de Juan. Espero obtener todo tipo de cosas de estas reuniones" (Bultmann \& Gogarten, 2002, p. 62).

${ }^{6}$ Para encontrar la esencia de lo que se dijo durante este seminario, consultaremos con provecho el artículo de Bultmann El problema de la ética en Pablo que se deriva directamente de él, aunque esto no implica no volver sobre las anotaciones originales (Bultmann, 1924).

${ }^{7}$ Carta de Rudolf Bultmann a Hans von Soden del 23 de deciembre de 1923 reproducida por Antje Bultmann Lemke en (Bultmann Lemke, 1984, p. 202). 
todos vamos a aprender como es debido"8. De la participación de Heidegger en este seminario, la historia ha conservado su exposición magistral sobre Das Problem der Sünde bei Luther, que se llevó a cabo durante dos sesiones consecutivas, la del 14 de febrero y la del 21 de febrero de 1924 (Heidegger, 1966, pp. 29-33). Sin embargo, según sugieren las fechas de las cartas citadas enviadas a von Soden y a Gogarten -el 22 y 23 de diciembre 1923-y como indican las anotaciones, Heidegger participó en un buen número de sesiones antes de hacer su exposición sobre Lutero. Además, ya se había hecho notar en el seminario unas semanas antes con una intervención sobre la Carta de San Pablo a los romanos. ${ }^{9}$

Esta intervención fue escrupulosamente transcrita por un estudiante llamado Martin Stallmann. Esta transcripción consta de tres páginas, las cuales han sido descuidadas hasta hoy por razones que verdaderamente no se explican. ${ }^{10}$ De hecho, tanto su tono como su contenido justifican sin dificultad el que nos interesemos en ellas. Estas páginas tienen, por supuesto, un valor documental, pero también un valor intrínseco por razones que se mostrarán con claridad a lo largo de su presentación.

La primera participación documentada de Heidegger en las discusiones tiene lugar en la octava sesión del seminario, que también es la primera del año 1924, la cual se realiza el 10 de enero de aquel año. Esta sesión se ajusta naturalmente a la temática del seminario: la ética de Pablo. ¿Cuál es la problemática exacta?

Para hacerlo simple, diremos que se trataba de la tensión entre el serjustificado y el ser-pecador, lo cual es también la tensión entre un indicativo -aquel que se ha hecho cristiano está efectivamente libre de pecado- y un imperativo aquel que se ha hecho cristiano, no obstante, se enfrenta siempre a poderes nefastos y debe tener cuidado de no obrar mal. ${ }^{11}$ Ahora bien, se debe señalar que en Pablo esta tensión a menudo se concentra en los mismos versículos. Bultmann aborda este problema mediante el desarrollo de una pregunta radicalmente diferente de la que domina la exégesis histórico-crítica (Baur, Holtzmann, Wernle, Weinel, Juncker Windisch): ${ }^{12 ~ “ ¿ C o ́ m o ~ l a ~ a n t i n o m i a ~ e n ~ l a s ~ d e c l a r a c i o n e s ~ d e ~ P a b l o ~ e s t a ́ ~ f u n d a d a ~ e n ~ l a ~}$

${ }^{8}$ Carta de Rudolf Bultmann a Friedrich Gogarten del 22 de diciembre de 1923 (Bultmann \& Gogarten, 2002, p. 53)

${ }^{9}$ A continuación, citaremos siempre esta carta paulina con las siglas Rm.

10 Sorprende, por ejemplo, el que Bernd Jaspert (Sachgemässe Exegese, 26-27), quien señala claramente su existencia, no se tome la molestia en hacerlas de conocimiento a los especialistas.

${ }^{11}$ Véase el Manuscrito 307a Nr. 3, 56. Comentamos el documento publicado en (Keiling, 2013, pp. 383-384). Sin embargo, conservamos la paginación del documento original conservado en los Archivos de la Universidad de Marburgo con el código UniA Marburg 307a Nr. 3. Usamos esta última fuente mediante la abreviatura "Ms" que hace referencia a "Manuscrito" y seguida de la página que corresponda.

${ }^{12}$ Las obras tratadas, discutidas y en su mayoría refutadas durante las sesiones anteriores del seminario son las siguientes: (Baur, 1864), (Holtzmann, 1897), (Wernle, 1897), (Weinel, 1911), (Juncker, 1904) y (Windisch, 1908). 
esencia de la cosa? En otras palabras, ¿es en la esencia del $\delta$ ¿ $\alpha \omega \theta$ cíc por lo que podemos hablar de su ser-justificado en la forma de un imperativo como en la de un realis?" (Ms 307a Nr. 3, 74). Respuesta: Hay para el creyente una "identidad entre don y acción" que no está enraizada en ningún otro lugar sino "en la fe" (Ms 307a Nr. $3,78)$. Creer es el acto propio del hacerse-cristiano, de modo que "los imperativos en $\mathrm{Rm} 6$ deben considerarse como aquellos que singularizan y describen la vida de un verdadero creyente" (Ms 307a Nr. 3, 78).

Es en este contexto que Heidegger interviene para hablar sobre "la vida del hombre en la fe" (Ms 307a Nr. 3, 78). Su punto de partida es Rm 6, 22 (Ms 307a Nr. 3, 79): "Pero ahora, libres del pecado y convertidos en siervos de Dios, lleváis los frutos que conducen a la santificación y su fin es la vida eterna". Este versículo "en

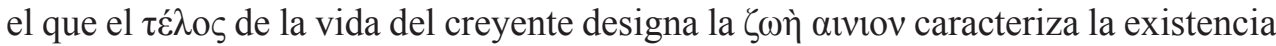
del cristiano como una existencia particular en la medida en que la finalidad de la vida no es otra que la vida misma, que el mismo vivir", comenta Heidegger (Ms 307a Nr. 3, 79). Aquí tenemos una primera indicación: la experiencia cristiana de la vida fáctica se define por una cierta tautología. Pero aquí esto no es una limitación. Lo que concede su especificidad a la vivencia cristiana es que se prueba a sí misma con la esperanza de acceder al sentimiento de la vida como tal. A lo que aspira no es otra cosa que a sí misma: apunta a su propia apropiación y, en cierto modo, a su auto-comprensión. Heidegger no niega que la vivencia cristiana tienda a dispersarse, pero su avance se realiza en un dispositivo temporal que enmarca la venida de Cristo y su retorno, dos acontecimientos que determinan incesantemente su presente mismo. En efecto, no dejemos de lado el contexto escatológico de Rm 6,22 y la sutileza que lo caracteriza. Si Pablo hace del mundo eterno la finalidad de la vida del creyente, nunca se detiene en el tema, prefiere centrar su propuesta en aquello que ha instaurado la posibilidad en primera instancia, a saber, la vida, la muerte y la resurrección de Cristo, y lo que marcará su llegada, es decir, la parusía. El "Día" significará el comienzo de un estar-siempre-con-el-Señor;"13 lo cual quiere decir que hasta entonces, el cristiano solo está, por así decir, intermitentemente con el Señor. La razón de esto radica en el pecado del que está liberado, pero que continúa tentándolo y lo empuja a dispersarse, es decir, a convertirse nuevamente en esclavo del mundo. Aun así, la existencia cristiana es su propio enemigo como también su propio camino de salvación; lo cual la vuelve absolutamente única en relación a las religiosidades que la rodean.

Por tanto, Heidegger deja de enfatizar su Eigenbeständigkeit y su carácter quasi-evidente: "esta finalidad de la vida no es de las que se relacionan artificialmente con la vida; ella está más bien allí en la fe" (Ms 307a Nr. 3, 79). La idea aquí es

${ }^{13}$ Véase 1 Ts 4, 17, de igual forma 1 Ts 2,$19 ; 3,13,4,15 ; 5,2 ; 5,23 ; 1$ Co 1,$8 ; 5,5 ; 15,23 ; 2$ Co 1 , 14; Flp 1, 6.10; 2, 16; Rm 2, 7; 5, 21; G1 6, 8. 
que la existencia cristiana lucha contra aquello en lo que se ha se convertido hic et nunc, como lo demuestra el "ahora" (vvvi) al principio de Rm 6, 22. El cristiano es creyente; la fe es, entonces, lo que él es, pero esta es tan buena cuanto él lo sea. También se aspira a poseerse en el ser con el fin de acceder a esta santificación" que hará de su existencia una "vida eterna en Jesucristo nuestro Señor" (Rm 6, 22-23). Si Heidegger confirma que Pablo "ve la existencia concreta del cristiano a partir de

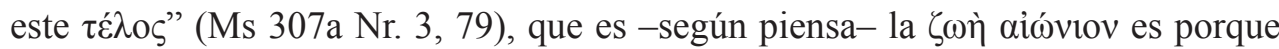
supone que sus oyentes conocen las sutilezas de la concepción neo-testamentaria del tiempo. A diferencia de los griegos, los proto-cristianos no distinguen entre tiempo y eternidad. Esta última es concebida por ellos, no como el resultado de la suspensión o abolición del tiempo, sino precisamente como el paso a otra era de tiempo infinito (Cullmann, 1947, p. 32 y p. 44). Oscar Cullmann explica sobre este tema que "en el Nuevo Testamento, el aî́v futuro es un futuro real, es decir, un futuro temporal. Hablar del aíóv que viene, sin mantener en todo su valor su carácter temporal, es una interpretación filosófica" (Cullmann, 1947, p. 46).

Ahora bien, esta última es precisamente contra la que Heidegger responde en su glosa a Rm 6, 22: aspira a la existencia concreta y temporal y no a aquella susceptible de ser llevada hacia un espacio fuera del tiempo, lo que es sinónimo de quietud. Esto se confirma tras la exposición en la que el filósofo explica que más allá del versículo tomado como ejemplo, la "conciencia que el cristiano tiene de sí mismo, de Dios y de las posibilidades de su obrar está caracterizada en Rm 6 como fe" (Ms 307a Nr. 3, 79). Ciertamente, no se exagera al decir que en realidad estos tres tipos de conciencia no son más que una misma conciencia, precisamente porque se unifican en la $\pi i ́ \sigma \tau ı \zeta$. El bautismo en la muerte de Jesucristo ha cambiado por completo la situación: ha destruido al hombre que residía en el hombre antes de dar una nueva vida que únicamente es posible en Dios y para él, y todo lo que se podrá hacer - $\mathrm{o}$ no hacer- será una manera de tomar posición respecto de Dios y de lo que hemos llegado a ser gracias a él. Por tanto, la fe se presenta como el denominador común de todas las disposiciones de la conciencia: el cristiano ya no vive sobre la base de una creencia virgen, sino a partir de una fe modalizada por el evento de Cristo. Para Heidegger, se sigue que "creer" no es un "acto, sino una modalidad del ser ante Dios" (Ms 307a Nr. 3, 79). Esta redefinición es particularmente poderosa, ya que está formulada en un registro ontológico y no solo en un registro dóxico. Creer compromete a todo el ser y no solo al corazón o al intelecto considerados tradicionalmente como los órganos de la vida espiritual. La fe lo compromete en su totalidad porque no hay nada en él que no esté afectado por su efectividad. Su forma de ser-en-el-mundo se revierte por completo; en otras palabras, se encuentra arrojado en un estado de crisis generalizada tan nuevo como irreversible. 
De este modo, parece que "la fe no es una propiedad", una cualidad o un atributo vulgar, "sino más bien lo que preocupa al cristiano en tanto que es" (Ms 307a Nr. 3, 79). Comprendamos que la fe es lo que acapara por completo la existencia del cristiano $\mathrm{y}$, por lo tanto, determina su relación práctica así como su relación teórica con el mundo e incluso, antes de eso, la forma en que está en el mundo o dentro del mundo. ${ }^{14}$ De igual forma es posible apoyarse en un sentido óntico de la noción de Besorgen para encontrar otra dimensión del sentido ontológico de la fe, en este caso el de proporcionar (a) la existencia cristiana. Dicho de modo literal: le da aquello que esta necesita imperiosamente, le procura lo que le es absolutamente necesario para sobrevivir. Pero recordemos sobre todo que es en el último de los primeros cursos de Friburgo realizados en el semestre de verano de 1923 donde Heidegger establece clara y distintamente que la Besorgen revela ser un síntoma de la disposición ontológica arraigada en nosotros con más profundidad que la Sorge. ${ }^{15}$ Ahora bien, es precisamente esta proposición la que encontramos aquí en una forma aún más elaborada: "Para el hombre, toda posibilidad de ser se mantiene en la preocupación" (Ms 307a Nr. 3, 79). Se podría pensar que el filósofo seculariza el modelo cristiano de la existencia, que extrae la estructura ontológica de un esquema óntico, pero parece más bien lo contrario, pues la proposición antes mencionada es puesta entre paréntesis y mientras se sale de ella se encuentra la fórmula clave, aquella según la cual "para el cristiano, la fe es lo que se mantiene en la preocupación” (Ms 307a Nr. 3, 79).

En la existencia creyente, la fe recubre perfectamente la preocupación -o a la inversa. Este es el "sentido de ser del cristiano" (Ms 307a Nr. 3, 79-80) que se puede extraer de los documentos originales del cristianismo primitivo. Ahora bien, Heidegger juzga que relacionada con este sentido, la significación del "imperativo" en Rm 6, "se hace evidente" (Ms 307a Nr. 3, 79-80). En términos concretos, esto significa que el imperativo no contradice el indicativo, sino que se inscribe en su extensión. Los versículos que mejor lo ilustran son, quizás, los de Rm 6, 10-11: "Porque muriendo $<$ Cristo $>$, es el pecado el que ha muerto de una vez por todas; viviendo se vive para Dios. De la misma manera vosotros, considerad que habéis muerto al pecado y vivís para Dios en Jesucristo". Pablo señala que lo que debería ser no es otra cosa que lo que ya es: la muerte al pecado y la vida en Cristo son realidades para aquellos que pueden ver con los “ojos del corazón” (Ef 1, 18), los cuales no son otros que los ojos

\footnotetext{
${ }^{14}$ Nos remitimos al $§ 12$ de Ser y Tiempo donde el filósofo lo volverá a decir con fuerza sintetizando los resultados de los primeros cursos de Friburgo y Marburgo.

${ }^{15}$ Véase (Heidegger, 1988; GA 63, p. 86 et passim). Este vínculo se anuncia desde el curso de semestre de invierno de 1921-1922 (Heidegger, 1985; GA 61, pp. 107, 110, 135-140, 184). Lo encontramos también en los cursos y textos del semestre de verano de 1922 (Heidegger, 2005b; GA 62, pp. 56 sq., 65 sq., 92, 144, 268, etc.) y (Heidegger, 2005a; GA 62, pp. 353 sq., 388 y 410). Por último, lo encontramos en el curso del semestre de invierno de 1923-1924 (Heidegger, 1994; GA 17, pp. 61, 105-107, 282-287, 317, 319).
} 
de la "fe" (2 Cor 5, 7). Ambos lugares bíblicos no son invocados en la exposición que estamos estudiando, pero se encuentran en una nota aislada de 1918 titulada "El carácter donador en el fenómeno de la fe". ${ }^{16}$ Lo que la fe ofrece es precisamente entender que no hay diferencia entre el indicativo y el imperativo, es decir que el

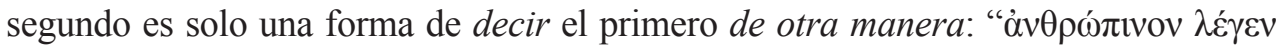

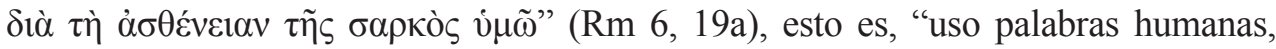
adaptadas a vuestra debilidad".

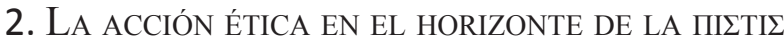

Dado que el problema del imperativo en Rm 6 se refiere principalmente a la parénesis -es al menos así como lo afronta Bultmann desde el comienzo de su seminario-, Heidegger trata de examinarlo directamente desde el punto de vista de la ética. Su razonamiento es muy simple: "En la medida en que todo obrar se sostiene en la fe, el obrar ético en tanto ético se muestra" (Ms 307a Nr. 3, 80). Creemos que el vocabulario utilizado no es inocente. Heidegger parece pensar bien sobre el proceso de mutación del sittliches Handeln en los términos de la Aufhebung hegeliana. En efecto, Rm 6 demuestra cómo Pablo afirma, niega y luego eleva -sobrepasa o superala acción ética en su dimensión ética. Siendo más precisos. En un primer momento, el Apóstol comienza reconociendo que la vida cristiana tiene sus imperativos, ya que exhorta a los creyentes a comportarse de cierta manera. En un segundo momento, niega que estos imperativos tengan un carácter imperativo en virtud de lo que él proclama, a saber, que los creyentes ya han muerto al pecado y viven para Dios en Jesucristo. En un tercer y último momento, rompe esta oposición al devolver a los cristianos a su fe: "Pero si hemos muertos con Cristo, creemos que también viviremos con él" (Rm 6, 8). En consecuencia, la acción ética ya no se entiende en el horizonte

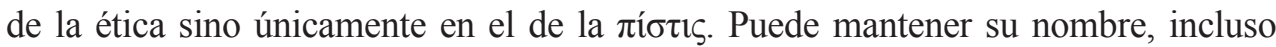
su apariencia, pero su sentido es transfigurado. Considerado "desde afuera", escribe Heidegger, el obrar del cristiano es un hecho ético" (Ms 307a Nr. 3, 80). Sin embargo, visto desde el interior, se ha convertido en algo más, esto es, en un hecho escatológico, ya que el obrar del cristiano consiste esencialmente en vivir en la espera de la parusía, es decir, en soportar (se) en la fe hasta la venida de Cristo. ${ }^{17}$

\footnotetext{
${ }^{16}$ Esta nota solo ha sido editada y publicada recientemente por Theodore Kisiel en (Kisiel, 2010, p. 320).

${ }^{17}$ Sobre este tema véase (Heidegger, 1995b; GA 60, p. 146) donde el filósofo evoca el cruce entre la "escatología" y la "facticidad" en el seno de los "heilsgeschichtlichen Fakten". Igualmente véase (Bultmann, 1966, p. 332): "Denn er <Christus > ist die eschatologische Tat Gottes, die alle Volksgeschichte als der Sphäre des Handeln Gottes mit den Menschen ein Ende macht". Quizás podamos adelantar que Heidegger encuentra allí el sentido original del termino Sitte -que Hegel no toma verdaderamente en consideración- como una manera de conducirse, es decir, un cierto modo de ser y, en este caso, de ser coram Deo.
} 
La interpretación heideggeriana de Rm 6 revela que la fe está lejos de referiste solo a la conciencia, ya que en el Corpus Paulinum más que en ningún otro lugar, la consciencia existe solo en virtud de un todo que la conduce a la existencia. De modo que Heidegger puede afirmar que "ser creyente abraza el ser del hombre en su totalidad" (Ms 307a Nr. 3, 80); lo cual equivale a decir que solo se puede creer auténticamente a condición de comprometerse sin descanso con una forma de vida, a condición de hacer de la fe la pre-comprensión de todo lo que se vive. Tengamos en cuenta que este proceso se lleva a cabo concretamente en una alianza entre actividad y pasividad. Según nuestro filósofo, "ser creyente significa" precisamente "dejar que cada situación sea dominada por la mirada hacia Dios" (Ms 307a Nr. 3, 80). Esta estructura parece corresponder a lo que la fenomenología de la religión más reciente llama atencionalidad, es decir, un modo de relación con uno mismo, con los demás, con el mundo y con Dios complementario pero distinto de la intencionalidad en lo que concierne precisamente das ganze Sein y no solo a das Bewusstsein. ${ }^{18}$ Aquí se habla de un abrirse integral a lo que es así como a lo que (ad) viene, de una receptividad que opera en un plano que subsume lo cognitivo y lo afectivo. Pues mirar hacia de Dios no solo es pensar en Dios ni tampoco sentirlo, sino vivir continuamente como si nada fuera posible sin Dios. ${ }^{19}$

Heidegger lo resume diciendo que ser creyente es, en última instancia, "ponerse-en-la-situación concreta" en la que solo se puede existir por medio de Dios (Ms 307a Nr. 3, 80). Precisemos: no solo a través de la mirada que dirigimos hacia Dios, sino en virtud de la atencionalidad antes mencionada, a través de la mirada que Dios mismo nos dirige ${ }^{20}$. Y subrayamos que la noción de Situation en la terminología proto-heideggeriana tiene un alcance fundamentalmente hermenéutico: la "situación" es para nosotros algo que pertenece al entender que realiza; pasa por lo que hace "la unidad de una multiplicidad", de modo que "no puede limitarse a un dominio particular del ser, ni siquiera proyectarse en la conciencia" (Heidegger, 1995b; GA 60, pp. 90, 92 y 91). Designa aquello en lo que el yo se encuentra irremediablemente intrincado, pero también en lo que es con más propiedad él mismo; por ello, es absolutamente "necesario entender la situación de Pablo para comprender los fenómenos" que operan en sus Cartas (Heidegger, 1995b; GA 60, p. 105). Por lo tanto, no hay dificultad en entender cómo Heidegger puede mantener que "todos los imperativos" mencionados en la Rm 6 apuntan en su "dirección" (Ms

\footnotetext{
${ }^{18}$ Véase (Depraz, 2008) y (Steinbock, 2007). Ambos autores se apoyan en parte en los escritos de Husserl sobre Percepción y Atención (Husserl, 2004).

${ }^{19}$ Aquí dos breves comentarios: 1) insistimos en la dimensión temporal de esta proposición hecha con el adverbio "continuamente"; 2) precisamos que el "como" en cuestión es fundamentalmente hermenéutico.

${ }^{20}$ Esta reciprocidad se acerca a la propuesta de la teología dialéctica, en concreta la de Barth propuesta en (Barth, 1919).
} 
307a Nr. 3, 80). Su objetivo es poner al cristiano frente al estado de cosas que se ha convertido en el suyo desde el evento de Cristo y la aceptación de la proclamación. Dado que su vida tiende a dispersarse, a desvanecerse ante sí misma $-\mathrm{y}$, por lo tanto, ante Dios- desdoblando su percepción de la realidad, los imperativos deben confrontar al hombre consigo mismo -y, por lo tanto, con Dios. Estos disimulan, por así decir, ángulos muertos en los que el cristiano se siente tentado a refugiarse contra el carácter insoportable de la angustia que le ha sido impuesta antes del Día. Al hacerlo, no crean nada ex nihilo, ni siquiera una obligación; se contentan con "alcanzar el ser del cristiano en la fe" (Ms 307a Nr. 3, 80).

Heidegger intenta demostrar lo que dice mediante el texto bíblico y, para esto,

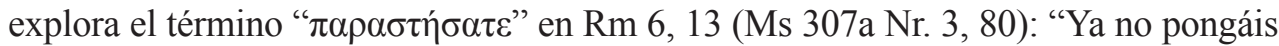
vuestros miembros al servicio del pecado como armas de injusticia, sino como vivientes resucitados de entre los muertos $\mathrm{y}$, con vuestros miembros como armas de justicia, poneros al servicio de Dios". Este último miembro se beneficia de una interpretación explicitante que profundiza sustancialmente su significación aparente. En efecto, nuestro filósofo lo volvía a traducir: "un juzgarse a sí mismo como puesto a disposición de Dios" (Ms 307a Nr. 3, 80). Ejemplo privilegiado del imperativo en Rm 6 , el $\pi \alpha \rho \alpha \sigma \tau \eta ́ \sigma \alpha \tau \varepsilon$ no indica una restricción que viene del exterior, sino una necesidad interior de reorientar la totalidad del sí mismo -y, por tanto, su atención- hacia lo que salva coincidiendo, a fin de cuentas, con el ser-cristiano en el que nos hemos convertido. La palabra clave en esta configuración parece ser la Hingabe. En efecto, Pablo ordena el abandono, al menos, en dos sentidos: abandonar definitivamente su vida anterior -al servicio de la injusticia- al darse cuenta de que estamos muertos al pecado; abandonarse a la voluntad de Dios que ha permitido esta transición hacia una nueva vida -al servicio de la justicia. Por lo tanto, no sorprende que tres años antes, Heidegger haya podido identificar el "abandono absoluto", el auténtico "amor de Dios", en el sentido más propio de la "fe" (Heidegger, 1995a; GA 60, p. 260).

Con claridad, el modo imperativo del disponerse de Rm 6, 13 es, de hecho, solo una variación en el modo indicativo del creer; únicamente sirve para decirlo de otra manera, puesto que se trata, en definitiva, de abandonarse a fin de comprenderse tal y como nos hemos ya convertido ante Dios o incluso en él (Cf. Heidegger, 1992; GA 58, p. 168). Téngase en cuenta que, si el sentido del imperativo está, por así decir, rodeado por la fe, entonces el creyente puede permitirse estar en cierta disposición sin tener ningún control sobre lo que le sucederá. El cristiano se dispone, pero es Dios quien dispone. Tanto el indicativo como el imperativo pertenecen a Dios y caen bajo su gracia. El hombre, por su parte, se pone a disposición del primero y se abandona a los efectos del segundo; de modo que "convertirse en creyente no significa crear nuevas posibilidades, sino aprovechar en todo momento la posibilidad de que el cristiano exista en la temporalidad" (Ms 307a Nr. 3, 80). 


\section{LA TRIDIMENSIONALIDAD DEL KAIPÓ $\Sigma$}

Aquí Heidegger utiliza un enfoque kairológico -en el sentido cristiano y no helénico-romano-helenístico-, de la proclamación paulina ${ }^{21}$. El Augenblick que se va a explotar no es un espacio infinito de libertad, sino una apertura que se cierra en un abrir y cerrar de ojos. Ahora bien, esta apertura, expresión paradójica de una contracción del tiempo, no es el lugar de un deber-ser sino simplemente el de un ser-allí, el de un existir. Ya es bastante difícil apropiarse de uno mismo en tales circunstancias, pues recordemos que el tiempo se acaba. El instante es ciertamente repetible, pero cada uno de sus acontecimientos marca un paso hacia el Final de los tiempos.

La situación continúa intensificándose y las dificultades se amplifican. A medida que los kaıpoí decretados por Dios se suceden, las posibilidades de ser uno mismo en la fe disminuyen. Sobre este tema citamos Rm 13, 11: "Además, ya sabéis en qué кaıрó salvación está võv más cerca de nosotros que cuando creímos la primera vez". Cada instante es único e irremplazable, no se puede desperdiciar ninguno para proyectarse fuera de esta hiero-temporalidad definida triplemente por el kaıpós pasado de la

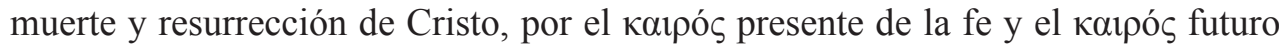
de la parusía. Este esquema ilumina de qué manera Heidegger argumenta que "la

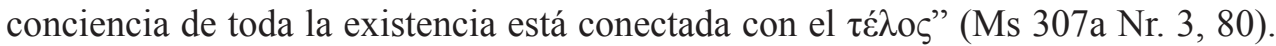
Según Pablo, el $\tau \dot{\lambda} \lambda o \varsigma$ solo puede ser Cristo. Ahora bien, él encarna precisamente los tres momentos antes mencionados: en el pasado como aquello que funda la fe, en el presente como aquello en lo que vive la fe, en el futuro como aquello que la fe espera febrilmente. Dicho esto, conectándose a Cristo, el cristiano no tiende a comprender lo incomprensible o a prever lo imprevisible, sino a realizarse en su presente que, al prolongarse, sigue siendo la única manera de vincularse con la temporalidad irregular a la que es conducido casi a pesar de él mismo. En efecto, en la medida en que se aproxima al Fin, el presente vivo se da como su finalidad privilegiada. Heidegger lo dice a su manera repitiendo que "el $\tau \dot{\lambda} \lambda$ os de mi vida es

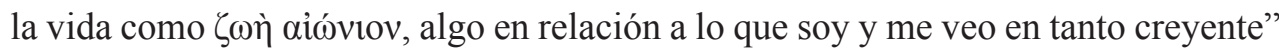
(Ms 307a Nr. 3, 80).

No hay otra solución que apoyarse en la propia vida para acceder a la vida eterna descrita anteriormente. Nuestro filósofo vincula los dos únicos versículos explícitamente mencionados hasta ahora: Rm 6, 22-23 y 6, 13. ¿Cuál es su objetivo? Demostrar que la apropiación de sí mismo en el presente se concentra finalmente en

\footnotetext{
${ }^{21}$ Heidegger con esto se inscribe en una prolongación de su introducción a la fenomenología de la religión del semestre de invierno de 1920-1921. Tendremos cuidado al comparar este curso con otros dos de los años 1921-1923 sobre Aristóteles a fin de ver correctamente la diferencia ente el кaıpós paulino y el Kaıpó griego - aristotélico, pero también estoico.
} 
la decidida alternativa entre la muerte y la vida. El salario del pecado es la muerte. Ahora bien, el cristiano ha muerto al pecado y, por tanto, se ha levantado de entre los muertos y está entre los vivos. De este modo, puede esperar la vida eterna. Pero, a fin de cuentas ¿lo va a entender? ¿Va a ser quien es en verdad por la gracia? ¿Se verá a sí mismo y vivirá como el creyente en el que se ha convertido? Esta problemática tiene repercusiones directas en la ética. La situación ad hoc de los proto-cristianos - tan cerca del evento pasado de la muerte y de la resurrección $y$ el evento futuro de la parusía- hace que el presente tenga una prioridad absoluta. No hay tiempo para la formación de nuevos mandamientos; apenas hay suficiente para aprovechar el kalpós de modo concreto a pesar de la presión intentando estar a la altura del indicativo establecido por Dios, es decir, vivir el tiempo creyendo, amando y obedeciendo.

Después del $\pi \alpha \rho \alpha \sigma \tau \eta ́ \sigma \alpha \tau \varepsilon$ en Rm 6, 13, Heidegger se detiene en el término

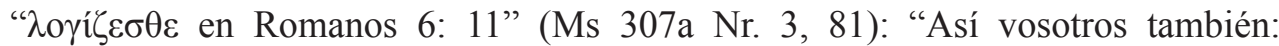
consideraos muertos al pecado y vivos para Dios en Cristo Jesús". Aquí nuevamente, quiere ser más preciso que la exégesis actual; lo que implica comenzar por destruir, es decir, excluir las interpretaciones de $\lambda$ o $\gamma$ í $\varepsilon \sigma \theta \varepsilon$ que no se ajusten al cumplimiento de la vivencia proto-cristiana. Entre ellas, el filósofo apunta dos en particular: por $\lambda$ oүí $\varepsilon \sigma \theta \varepsilon$, Pablo "no aspira a una decisión teórica o una disputa cogitativa" (Ms 307a Nr. 3, 81). Vivir para Dios en Jesucristo no es un estado fundado por una discusión y argumentos, como si uno necesitara ser convencido o convencerse racionalmente de que se debe morir al pecado para renacer. Esta experiencia no es la consecuencia de un juicio constituido (En contra de Jülicher, 1907, p. 36), es el resultado de una meditación. Esta no tiene que luchar para imponerse al creyente porque se ofrece a él inmediatamente como un don gratuito de Dios. Para aceptarlo, el cristiano no tiene que reflexionar, sino simplemente tenerlo en cuenta, es decir, tomar conciencia de ello y ponerlo en su haber (2 Cor 10: 7, Fil 4, 8). Es respecto a esto por lo que Heidegger puede después establecer el $\lambda \circ \gamma i \zeta \varepsilon \sigma \theta \varepsilon$ positivamente como "la conciencia específica que posee el hombre en tanto que se trata a sí mismo prácticamente, se interroga a sí mismo y se comunica consigo mismo" (Ms 307a Nr. $3,81)$. Se trata de moverse, vivirse, verse y comprenderse en la situación concreta única cuyos contornos han sido esbozados antes: el creyente está llamado a ser uno de los actores determinantes de una historia de la salvación a punto de conocer un giro mayor e incluso decisivo.

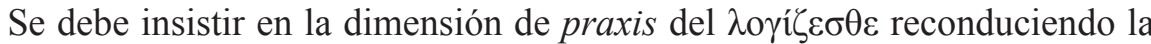
declaración paulina a la alternativa entre creer y no creer, que es una decisión que solo puede tomarse en su fuero interno no sobre la base de una demostración lógica, sino de una sumisión activa al estado de las cosas por poco que aceptemos reconocerlo. Heidegger hace eco de esto al hablar de un "ser circunspecto frente a lo que hago" 
(Ms 307a Nr. 3, 81). No veamos aquí una recuperación de la prudencia reflexiva,

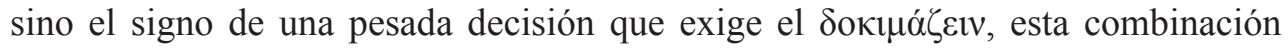
improbable del examen sabio y la inspiración espontánea que resulta de la absorción de nuestra voluntad en la de Dios (Cullmann, 1947, pp. 164-165).

En su Einleitung in die Phänomenologie der Religion de 1920-1921 (GA

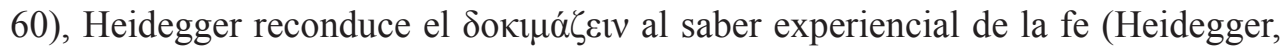
1995b; GA 60, p. 113). Es precisamente este carácter experiencial que el filósofo

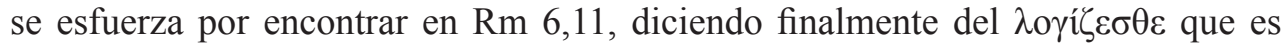
"cumplimiento explicitante de la conciencia creyente" (Ms 307a Nr. 3, 81). La fe no es ni un pensamiento ni un grito, sino una forma del vivir-se y del comprenderse concretamente frente a algo que nos supera -ya sea que situemos este algo, este Otro o este Todo-Otro, Dios, dentro o fuera de la conciencia. La fe apela a la clarificación de su situación. Ahora bien, si esta clarificación puede realizarse a través de palabras, el lenguaje que las lleva solo traduce una manera de ser-sí-

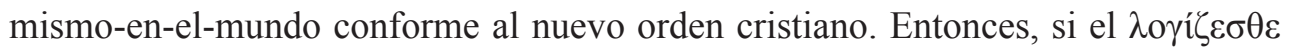
apunta a la conciencia solo puede tratarse de la conciencia de cumplimiento en el seno de una existencia (Da-sein) que es consecuencia de la conciencia vivida de la fe adquirida en virtud de la aceptación de la proclamación. De modo que Heidegger puede declarar que "la fe es al mismo tiempo la conciencia específica que el cristiano tiene de sí mismo, de modo que la fe también domina la conciencia de sí" (Ms 307a Nr. 3, 81). Lo que distingue al cristiano de sus contemporáneos es que su relación con el mundo excluye suprimir su relación consigo mismo. Dado que su mundo de la vida (Lebenswelt) tiene su centro de gravedad en su propio mundo (Selbstwelt) que también es el mundo de sus experiencias internas, en primer lugar, las de la fe (Heidegger, 1992; GA 58, p. 61). ¿Hay algo más normal que esto?

\section{CONCLUSIÓN}

La caída de la exposición de Heidegger es dramática y enigmática. Sus desarrollos sobre la vida del hombre en la fe en Rm finalmente llevan al filósofo a declarar que "se puede comprender toda la actitud en el capítulo 6 únicamente como lector creyente" (Ms 307a Nr. 3, 81). Esta afirmación debe leerse sobre dos planos distintos, pero complementarios.

El primer plano hace referencia al problema de la empatía (Cf. Heidegger, 1995b; GA 60, pp. 85 y 88-90). ${ }^{22}$ No se puede pretender acceder al sentido de las

\footnotetext{
${ }^{22}$ Heidegger reconoce la extrema dificultad de empatizar con Pablo y trata de trazar un camino entre dos extremos: por un lado, los que se mantienen alejados de la situación en aras de la objetividad científica a razón de que el mundo ambiente de Pablo ya no nos es accesible y, por otro lado, los que fantasean y se entusiasman demasiado con el Apóstol, haciendo desaparecer esta distancia crítica sin la cual ninguna comprensión en sentido propio es posible.
} 
palabras paulinas a menos que se traslade a la situación de Pablo el sentido preciso de realizar con él la escritura de la Carta (Cf. Heidegger, 1995b; GA 60, pp. 87 y $100)$, es decir, volver sobre el camino motivacional que lo ha llevado a proclamar lo que proclama y cómo lo proclama. Aquí estamos en los límites de la comprensión fenomenológica en el sentido de que nada separa la fusión de horizontes de la confusión. El segundo plano hace eco correspondientemente a la pregunta sobre la posibilidad misma de comprender filosóficamente el Nuevo Testamento que persigue a Heidegger desde la afirmación ambigua del a-teísmo de la filosofía en $1921 .{ }^{23} \mathrm{Se}$ tendría que creer para comprender, pero comprender de modo completo requeriría un cuestionamiento radical que la fe puede no ser capaz de soportar. Dicho esto, es probable que al hacer en sí misma la prueba concreta de esta paradoja, el filósofo se vuelva capaz de decir algo esencial sobre la religiosidad proto-cristiana.

Qué se puede concluir, si no que la historia de la relación entre Heidegger y Bultmann se origina sin lugar a dudas en el seminario sobre la ética de Pablo. Dicho de otro modo, que el diálogo más fructífero entre la filosofía y la teología del siglo XX se llevó a cabo por primera vez en torno a la exégesis de las Escrituras. Este dato inspirará nuestro comentario final según el cual las relaciones entre fenomenología y teología a partir de ahora ya no podrían conocer desarrollos sustanciales a menos de que hagan un Schritt zurück a fin de comprender en un doble sentido: como un retorno a lo que se juega históricamente, incluso historialmente, en este encuentro inaugural entre Heidegger y Bultmann en torno a la exégesis, y como un retorno a la exégesis bíblica en sí misma, que es la única que posee la clave sistemática de un pacificación y una colaboración fructífera en tanto que responde a las cuestiones hermenéuticoexistenciales yendo por encima de toda dogmática filosófica o teológica.

\section{BIBLIOGRAFÍA}

Barth, Karl. (1919). Der Römerbrief. Bern: Bäschlin.

Baur, Ferdinand Christian (Ed.). (1864). Vorlesungen über neutestamentliche Theologie. Leipzig: Fues.

Bultmann Lemke, Antje. (1984). Der unveröffentlichte Nachlasse Rudolf Bultmann Ausschnitte aus dem biographischen Material. In Bernd Jaspert (Ed.), Rudolf Bultmanns Werk und Wirkung. Darmstadt: Wissenschaftliche Buchgesellschaft.

Bultmann, Rudolf. (1924). Das Problem der Ethik bei Paulus. Zeitschrift für die neutestamentliche Wissenschaft und die Kunde der älteren Kirche, 23, 123-140.

${ }^{23}$ Véase (Heidegger, 1985; GA 61, p. 197) y (Heidegger, 2005a; GA 62, p. 28). 
Bultmann, Rudolf. (1966). Glauben und Verstehen (6 ed.). Tübingen: J.C.B. Mohr.

Bultmann, Rudolf, \& Gogarten, Friedrich. (2002). Briefwechsel 1921-1967. Tübingen: Mohr Siebeck.

Camilleri, Sylvain. (2017). Heidegger et les grandes lignes dune phénoménologie herméneutique du christianisme primitif. Dordrecht: Springer.

Cullmann, Oscar. (1947). Christ et le temps. Temps et histoire dans le christianisme primitif. Paris/Neuchâtel: Delachaux/Niestlé.

Depraz, Natalie. (2008). Le corps glorieux. Phénoménologie pratique de la Philocalie, des Pères du Désert et des Pères de l'Église. Leuven: Peeters.

Gadamer, Hans-Georg. (1977). Philosophische Lehrjahre. Eine Rückschau. Frankfurt am Main: Klostermann.

Heidegger, Martin. (1966). Das Problem der Sünde bei Luther. Protokoll von N.N. y Heinrich Schlier. In Bernd Jaspert (Ed.), Sachgemässe Exegese. Die Protokolle aus Rudolf Bultmanns Neutestamentlichen Seminaren 1921-1951 (pp. 29-33). Marburg: Elwert.

Heidegger, Martin. (1977). Sein und Zeit. In F.-W. von Herrmann (Ed.), Heidegger Gesamtausgabe 2. Frankfurt am Main: Klostermann.

Heidegger, Martin. (1985). Phänomenologische Interpretationen zu Aristoteles. Einführung in die phänomenologische Forschung (WS 1921/22). In Walter Bröcker \& Käte BröckerOltmanns (Eds.), Heidegger Gesamtausgabe 61. Frankfurt am Main: Klostermann.

Heidegger, Martin. (1988). Ontologie. Hermeneutik der Faktizität (SS 1923). In Käte Bröcker-Oltmanns (Ed.), Heidegger Gesamtausgabe 63. Frankfurt am Main: Klostermann.

Heidegger, Martin. (1992). Grundprobleme der Phänomenologie. In Hans-Helmuth Gander (Ed.), Heidegger Gesamtausgabe 58. Frankfurt am Main: Klostermann.

Heidegger, Martin. (1994). Einführung in die phänomenologische Forschung. In F.-W. von Herrmann (Ed.), Heidegger Gesamtausgabe 17. Frankfurt am Main: Klostermann.

Heidegger, Martin. (1995a). Augustinus und der Neuplatonismus. In Matthias Jung, Thomas Regehly \& Claudius Strube (Eds.), Heidegger Gesamtausgabe 60. Frankfurt am Main: Klostermann.

Heidegger, Martin. (1995b). Einleitung in die Phänomenologie der Religion (WS 1920/21). In Matthias Jung, Thomas Regehly \& Claudius Strube (Eds.), Heidegger Gesamtausgabe 60. Frankfurt am Main: Klostermann.

Heidegger, Martin. (2005a). Anzeige der hermeneutischen Situation. In Günther Neumann (Ed.), Heidegger Gesamtausgabe 62. Frankfurt am Main: Klostermann.

Heidegger, Martin. (2005b). Phänomenologische Interpretationen ausgewählter Abhandlungen des Aristoteles. In Günther Neumann (Ed.), Heidegger Gesamtausgabe 62. Frankfurt am Main: Klostermann.

Heidegger, Martin, \& Bultmann, Rudolf. (2009). Briefwechsel 1925-1975. Frankfurt am Main/Tübingen: Klostermann/Mohr Siebeck. 
Heidegger, Martin, \& Jaspers, Karl. (1990). Briefwechsel 1920-1963. Frankfurt am Main: Piper.

Holtzmann, Heinrich Julius. (1897). Lehrbuch der neutestamentliche Theologie. Tübingen: J.C.B. Mohr.

Husserl, Edmund. (2004). Wahrnehmung und Aufmerksamkeit. Texte aus dem Nachlass (1893-1912). In Thomas Vongehr \& Regula Giuliani (Eds.), Husserliana: Edmund Husserl Gesammelte Werke XXXVIII. Dordrecht: Springer.

Jülicher, Adolf. (1907). Der Brief an die Römer. In Johannes Weiss (Ed.), Die Schriften des Neuen Testaments (Vol. II). Göttingen: Vandenhoeck \& Ruprecht.

Juncker, Alfred. (1904). Die Ethik des Apostels Paulus (Vol. I). Halle: Niemeyer.

Keiling, Tobias (Ed.). (2013). Heideggers Marburger Zeit. Themen, Argumente, Konstellationen. Frankfurt am Mein: Klostermann.

Kisiel, Theodore. (2010). Notes for a Work on the 'Phenomenology of Religious Life' (1916-1919). In Sean J. McGrath \& Andrezj Wiercinski (Eds.), A Companion to Heidegger's Phenomenology of Religious Life. Amsterdam/New York: Rodopi.

Steinbock, Anthony J. (2007). Phenomenology \& Mysticism. The Verticality of Religious Experience. Bloomington: Indiana University Press.

Weinel, Heinrich. (1911). Biblische Theologie des neuen Testaments. Die Religion Jesu und des Urchristentums. Tübingen: J.C.B. Mohr.

Wernle, Paul. (1897). Der Christ und die Sünde bei Paulus. Freiburg/Leipzig: J.C.B. Mohr. Windisch, Hans. (1908). Taufe und Sünde im ältesten Christentum bis auf Origenes. Ein Beitrag zur altchristlichen Dogmengeschichte. Tübingen: J.C.B. Mohr. 